\title{
A prática do auto-exame da mama em mulheres de baixa renda: um estudo de crenças
}

\author{
Suy-Mey C. de Mendonça Gonçalves \\ Mardonio Rique Dias \\ Universidade Federal da Paraíba
}

Resumo

Tem sido objeto de preocupação da literatura médica e, mais recentemente, da psicológica, a alta prevalência do câncer de mama nas mulheres. O diagnóstico precoce muito favorece na redução dos índices e, entre os meios de detecção, o "auto-exame da mama" se caracteriza como uma prevenção secundária, sem custos e segura. Pesquisas demonstram, contudo, que a prática do auto-exame da mama é insatisfatória, principalmente em mulheres de escolaridade e nível sócio-econômico baixos. Segundo a Teoria da Ação Racional (TRA), a maioria dos comportamentos humanos pode ser explicada em termos de crenças comportamentais ou normativas. Nesse sentido, a presente pesquisa teve por objetivo investigar as crenças modais salientes desse estrato populacional, desprovido de informações claras e serviços de saúde efetivos, através de um levantamento em uma amostra de 40 mulheres entrevistadas em ambulatórios públicos de João Pessoa (PB). As entrevistas continham itens sobre as vantagens e desvantagens do auto-exame da mama, conhecimentos e dados sócio-demográficos. A média de idade da amostra foi de 32,5 anos $(\mathrm{DP}=11,37)$. As entrevistas indicaram um total de 132 crenças comportamentais, que foram categorizadas em nove dimensões e 166 crenças normativas, categorizadas em quatro dimensões. Estes resultados demonstraram que as crenças influenciam a adesão à prática do auto-exame da mama e servirão de base para 
142 S. C. de M. Gonçalves e M. R. Dias

que os profissionais que lidam com campanhas públicas de prevenção voltem sua atenção para esses aspectos e abordem conteúdos que promovam não só a prática do auto-exame da mama, mas outras estratégias preventivas nesta significativa população.

Palavras-chave: auto-exame da mama, baixa renda, crenças, prevenção
Key-words:

\begin{abstract}
The practice of breast self-examination among low-income women: a study of beliefs

It has been object of concern to the medical literature and, more recently, to psychology, the high prevalence of breast cancer in women. The early diagnosis helps to decrease the index, and among the methods for detection, the "breast selfexamination" appears as a secondary prevention, secure and without costs. Researches have shown, however, that the practice of breast self-examination is not satisfactory, mainly to women of a low schooling as well as a low social-economic level. According to the Theory of Reasoned Action (TRA), most of human behavior can be explained in terms of behavioral or normative beliefs. So, the present research aims to investigate the prominent modal beliefs of low income women, who lack clear information and effective health services, by using a sample of 40 women interviewed at a public hospital in João Pessoa (PB, Northeast of Brazil). The interviews contained items about the advantages and disadvantages of the breast self-examination, knowledge and sociodemographic data. The average age of the sample was 32,5 years $(\mathrm{DP}=11,37)$. Results indicate a total of 132 behavioral beliefs, which were classified into 9 dimensions and 166 normative beliefs, classified into 4 dimensions. These results demonstrate that beliefs do influence adherence to a practice of breast self-examination, and might make public prevention campaigns to turn their attention to these aspects, promoting not only the practice of breast self examination, but other preventive strategies for this significant portion of the population.
\end{abstract}


$\mathrm{N}$ as últimas décadas, o câncer de mama tem sido um dos maiores problemas de saúde pública em todo o mundo. Em termos epidemiológicos, o câncer de mama é o tumor de maior incidência nos Estados Unidos, Canadá, Noruega, Cuba, Índia, Suécia, Austrália e Brasil, e o segundo no Equador, Argélia e Japão (Lopes, Rebelo, Adib \& Abreu, 1996). Nos Estados Unidos, a Sociedade Americana de Câncer (American Cancer Society, 1993) estimou que, em 1992, 180.000 mulheres contraíram o câncer de mama e que, com um aumento de $4 \%$ ao ano, chegaremos ao ano $2000 \mathrm{com}$, aproximadamente, 225.000 casos novos e um total de um milhão de casos novos em todo o mundo.

Percebe-se que o número de casos novos aumenta cada vez mais e que a projeção desta incidência é ainda maior nos países em desenvolvimento, em relação aos países desenvolvidos. Isto é explicado, principalmente, devido à falta de programas adequados de rastreamento nestes países (Bland \& Copeland, 1994).

A recente discussão em torno dos perigos e da incidência do câncer de mama enseja, particularmente no Brasil, uma preocupação devido a este já ter superado em incidência o câncer do colo do útero, anteriormente, o de maior incidência nas neoplasias femininas; e uma reflexão sobre o preconceito, a desinformação das mulheres em relação à doença e à importância dos procedimentos de detecção precoce.

O Serviço de Epidemiologia do Pró-Onco (Coordenação de Programas de Controle de Câncer - Ministério da Saúde), estimou para o ano de 1998, 32.695 novos casos de câncer de mama em todo o país, ocupando o primeiro lugar das neoplasias nas mulheres, o primeiro lugar das neoplasias gerais entre homens e mulheres, e o primeiro lugar de mortalidade feminina, com 7.165 casos novos de óbitos esperados. Estas estimativas são seguidas por doenças do aparelho circulatório, doenças infecciosas e parasitárias, doenças endócrinas nutricionais e do aparelho respiratório, ficando o câncer de mama com o terceiro lugar de mortalidade por cânceres entre homens e mulheres. Na Tabela 1 pode-se observar, de acordo com as três últimas estimativas de incidência e mortalidade do câncer de mama no Brasil, que há um aumento constante dos índices nas duas categorias. 
144 S. C. de M. Gonçalves e M. R. Dias

Tabela 1

Incidência e mortalidade de câncer de mama - Brasil 1966-1998

\begin{tabular}{ccc}
\hline Anos & Incidência & Mortalidade \\
\hline 1996 & 31.210 & 6.450 \\
1997 & 28.310 & 6.780 \\
1998 & 32.695 & 7.165 \\
\hline
\end{tabular}

Fonte: Estimativa da Incidência e Mortalidade por Câncer- Ministério da Saúde $1996 / 1997 / 1998$

Ainda, segundo dados do Ministério da Saúde, a região Sudeste, onde se localizam as grandes metrópoles brasileiras, abriga, incontestavelmente, o maior número de incidência (12.885), seguida das regiões Nordeste (8.770), Sul (8.250) e Centro-oeste (1.710). Na região Norte, o câncer de mama ocupa o segundo lugar, precedido pelo câncer do colo do útero. As causas desta incidência elevada em nosso país são explicadas pela questão histórica da urbanização (processo de desenvolvimento da industrialização), questões culturais (hábitos de vida) e questões sócio-políticas, como a ausência de programas oficiais efetivos para o controle da doença e a deficiência dos serviços de saúde destinados à saúde da mulher.

Segundo especialistas, não se pode fazer uma prevenção primária ao câncer de mama, pois esta só acontece quando se estabelece uma relação clara entre a doença e seus agentes causadores (ex.: fumar e câncer no pulmão). No caso do câncer de mama, os agentes causadores da doença são ainda desconhecidos, e a única ação efetiva que se tem é uma "prevenção secundária" em termos de diagnóstico em estágios iniciais da doença. Estudos demonstram que a detecção precoce, efetivamente, leva a uma maior sobrevida das mulheres com câncer de mama ( Foster Jr. et al., 1978; Greenwald, Nasca \& Lawrence, 1978; Celentano \& Holtzman, 1983; Huguley Jr. \& Brown, 1981 e Salazar, 1994).

Entre os meios de detecção precoce ao câncer de mama temos: - o "exame sistemático da mama", feito pelo profissional especializado; 
- a "mamografia", que seria um exame radiológico, de alta precisão e custo igualmente elevados, dificultando o acesso da população de menor poder aquisitivo;

- o "auto-exame da mama" que é o exame feito pela própria mulher.

Dentre estes meios de detecção precoce, o auto-exame da mama, apesar de não possuir a mesma eficácia que as técnicas mamográficas ou profissionais, é considerado como o principal método de detecção do câncer de mama pelas mulheres, já que na maioria das vezes, é a própria mulher quem encontra o tumor. Através do auto-exame da mama, a mulher pode detectar pequenas mudanças nas propriedades físicas das mamas, o que a leva a descobrir nódulos com $1 \mathrm{~cm}$ de diâmetro, diminuindo assim a probabilidade de metástase e aumentando a sua sobrevida.

Embora o conhecimento das vantagens do auto-exame da mama seja bastante divulgado pelos meios de comunicação, setores educacionais e programas de saúde pública, diversos estudos demonstram que o índice de prática é incipiente em diversas partes do mundo (Foster Jr. et al., 1978; Celentano \& Holtzman, 1983; Huguley Jr. \& Brown, 1981). Outros estudos sobre fatores sócio-econômicos que influenciam a prática do auto-exame da mama, mostram que as mulheres de maior nível educacional e de renda são as de maior adesão e que detêm maiores conhecimentos sobre o auto-exame da mama (Howe, 1981; Edwards, 1980; Dela Coleta, 1992; Freitas Jr., Soares, Melo, Andrade \& Philocréon, 1996; Dias da Costa, Piccini \& Moreira, 1995; Thomas \& Fick, 1995 e Seidl \& Gimenes, 1997).

Tais estudos reforçam a percepção de que: 1) as mulheres de baixo estrato social, educacional e informacional são as que mais precisam serem persuadidas a praticar o auto-exame da mama; e 2) há necessidade premente de se estudar os fatores psicossociais que determinam esta não-adesão, uma vez que as campanhas veiculadas pela mídia são ineficazes, com informações que não expressam o universo de crenças dessas mulheres sobre o comportamento em questão.

Revendo na literatura estudos que relacionam as crenças como determinantes do comportamento de praticar o auto-exame da mama, independentemente do modelo teórico utilizado (Hill, Gardner \& 
Rassaby, 1985; Calnan \& Rutter, 1986; Rutledge, 1987; Champion, 1985; Lierman, Young, Kasprzyk \& Benoliel, 1990, Lierman, Young, Powell-Cope, Gergiadou \& Benoliel, 1994; D'Amorim, Freitas \& de Sá, 1992; Yoshioca \& de Souza, 1994; e Baron, Houfek \& Foxall, 1997), percebe-se que a maioria deles leva em conta as diversas faixas de renda, não existindo estudos específicos na população feminina de baixa renda e de baixa escolaridade.

Isto, em termos de Brasil, é preocupante, tendo em vista que a grande maioria da população brasileira é formada por pessoas de nível sócio-econômico, educacional e cultural extremamente baixos que, além da dificuldade em ter acesso a atendimento público, geralmente chegam a procurar ajuda especializada em estágios tardios da doença. Estudos comprovam que aquelas que retardam a ida ao médico especializado são mulheres mais velhas, de classe social e educação mais baixas (Cameron \& Hilton, 1968; Costa, Mogos \& Toma, 1985 e Willians, 1976 apud Bland \& Copeland, 1994).

Dentro do raciocínio de que a prática do auto-exame da mama pode ajudar, e muito, a reverter o quadro da doença no país, e que isto está correlacionado com o processo cognitivo de influência da informação para a aquisição de novos repertórios de crenças e pensamentos que tenham a finalidade de uma mudança comportamental numa direção mais preventiva, optou-se pelo uso da Teoria da Ação Racional (Fishbein \& Ajzen, 1975; Ajzen \& Fishbein, 1980), uma vez que esta teoria tem uma evidência empírica considerável na explicação e predição de vários comportamentos preventivos e individuais de saúde ( Hill et al., 1985; D’Amorim \& Gomide, 1986; Fishbein, 1990; Lierman et al., 1990, 1994; Brubaker \& Fowler, 1990; Dias, 1995, Saldanha, 1997).

Segundo os autores da "Teoria da Ação Racional" (TAR) (Fishbein \& Ajzen, 1975; Ajzen \& Fishbein, 1980), é a intenção comportamental o melhor preditor da probabilidade de a pessoa executar ou não o comportamento. O comportamento desempenhado se dá de forma racional, ou seja, as pessoas enquanto seres humanos racionais, utilizam sistematicamente as informações adquiridas para atingir tal comportamento. 
A intenção comportamental, por sua vez, tem dois determinantes básicos que são a atitude comportamental e a norma subjetiva. A atitude seria o fator pessoal e a norma subjetiva seria o fator social (Dias, 1995). Tanto a atitude quanto a norma subjetiva possuem subcategorias que são: as crenças comportamentais, relativas ao que a pessoa crê que irá acontecer por desempenhar o comportamento e as avaliações das conseqüências deste comportamento; e as crenças normativas, originadas das pressões sociais de seus referentes (família, amigos, sociedade) e a motivação para concordar com esses referentes. A Figura 1 apresenta, esquematicamente, o modelo da Teoria da Ação Racional.

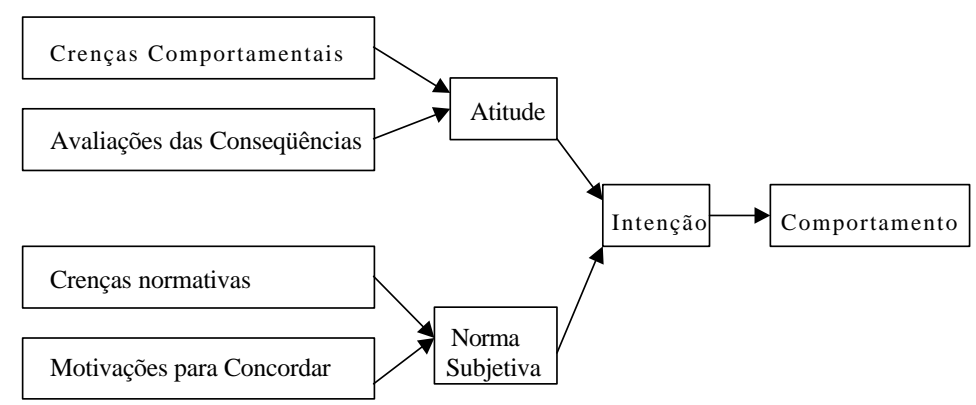

Figura 1: Teoria da Ação Racional de Fisbein e Ajzen (1975). Adaptada de Dias (1995).

Os autores afirmam ainda que, objetivando-se mudar um determinado comportamento numa população específica, faz-se necessário conhecer os determinantes deste comportamento e verificar também se o comportamento a ser investigado está sob o controle volitivo do indivíduo, ou seja, o indivíduo possui controle real sobre o comportamento, não dependendo de outras pessoas ou serviços de saúde para executá-lo, tais como, exame do câncer do colo do útero, mamografia, uso de preservativos etc. A prática do auto-exame da mama configura-se, assim, como de controle volitivo, sem precisar de nenhuma locomoção, nem serviços ou profissionais de saúde. 


\section{Método}

A pesquisa, com base no modelo metodológico dos autores da TAR, objetivou identificar as crenças e referentes modais salientes das mulheres de baixa renda relativas ao comportamento alvo, "praticar o auto-exame da mama".

\section{Sujeitos}

A amostra entrevistada constou de 40 mulheres, usuárias de um ambulatório do Sistema Único de Saúde (SUS), na cidade de João Pessoa (PB), com idade entre 17 a 60 anos, e com uma média de 32,5 $\operatorname{anos}(\mathrm{DP}=11,37)$.

A maioria (60\%) residia em João Pessoa e as demais no interior. Na situação conjugal civil, há um predomínio das mulheres casadas, regularmente ou consensualmente $(67,5 \%)$, em detrimento das solteiras $(27,5 \%)$, das viúvas e das separadas (2,5\%). O nível de escolaridade é bastante baixa, visto que $40 \%$ não chega a ter o primeiro grau completo, $35 \%$ tem um nível educacional médio, $22,5 \%$ possui o segundo grau completo e apenas $10 \%$ tem curso superior. Quanto à profissão/ocupação, $55 \%$ da amostra só exerce atividades em casa e $45 \%$ trabalha fora, em atividades que variaram de empregada doméstica a professora primária. No que se refere à renda familiar, a maioria, situa-se na faixa de um a dois salários mínimos, com 55\% da amostra não possuindo renda própria, e as que tem, recebendo no máximo dois salários mínimos. Em relação ao número de filhos, $45 \%$ da amostra tinha de um a dois filhos, $20 \%$ de três a quatro filhos, $5 \%$ acima de cinco filhos e $30 \%$ não tinha filhos. A idade da primeira gestação se concentrou dos 16 aos 19 anos de idade, fator considerado de risco ao câncer de mama. Por fim, quanto à religião, observou-se que $82,5 \%$ da amostra era composta de católicas, $15 \%$ de evangélicas e 2,5\% de Testemunhas de Jeová.

Os dados da Tabela 2 demonstram que a maioria das mulheres entrevistadas já ouviu falar do câncer de mama (85\%), é ciente de que ele pode matar (95\%), e igualmente acredita que ele pode ser curável (70\%).

Embora conheçam o câncer de mama, um percentual alto da amostra (45\%) não conhece nenhuma forma de evitá-lo. E um fato 
Tabela 2

Conhecimentos do Câncer de Mama e do Auto-exame $(N=40)$

\begin{tabular}{|c|c|c|}
\hline & Frequiência & $\%$ \\
\hline \multicolumn{3}{|c|}{ Ouviu falar do câncer de mama? } \\
\hline sim & 34 & 85 \\
\hline não & 6 & 15 \\
\hline \multicolumn{3}{|c|}{ Sabe que o câncer mata? } \\
\hline sim & 38 & 95 \\
\hline não & 2 & 5 \\
\hline \multicolumn{3}{|c|}{ O câncer de mama é curável? } \\
\hline $\operatorname{sim}$ & 28 & 70 \\
\hline não & 12 & 30 \\
\hline \multicolumn{3}{|c|}{ Conhece alguma forma de evitá-lo? } \\
\hline $\operatorname{sim}$ & 22 & 55 \\
\hline não & 18 & 45 \\
\hline \multicolumn{3}{|c|}{ Conhece o auto-exame da mama? } \\
\hline $\operatorname{sim}$ & 24 & 60 \\
\hline não & 16 & 40 \\
\hline \multicolumn{3}{|c|}{ Sempre vem ao ginecologista? } \\
\hline $\operatorname{sim}$ & 22 & 55 \\
\hline não & 14 & 35 \\
\hline às vezes & 4 & 10 \\
\hline \multicolumn{3}{|c|}{ Alguma vez já foi examinada na mama? } \\
\hline $\operatorname{sim}$ & 16 & 40 \\
\hline não & 24 & 60 \\
\hline \multicolumn{3}{|l|}{ Hábitos gerais } \\
\hline fuma & 11 & 29 \\
\hline bebe & 3 & 8 \\
\hline drogas & 3 & 8 \\
\hline come gorduras & 21 & 55 \\
\hline
\end{tabular}

preocupante e já encontrado em pesquisas anteriores (Freitas Jr. et al., 1996; Dias da Costa, Piccini \& Moreira, 1995) foi que, apesar de $60 \%$ das mulheres conhecer o auto-exame e portanto, ser maioria, $40 \%$ das mulheres não o conhecia; ou seja, as que não o conhecem, são totalmente desinformadas do que seja, e para que serve o autoexame da mama. Pode-se também afirmar, de acordo com pesquisas anteriores, que o baixo nível educacional da amostra é uma das causas da falta de conhecimento e de prática desta parcela populacional. Constatou-se, também, que apenas $38 \%$ da amostra tinha sido exami- 
nada nas mamas e que $62 \%$ nunca havia sido examinada. Das que foram examinadas, $17,5 \%$ foi decorrente do período gestacional, 7,5\% por dores na mama e 2,5\% devido à consulta médica. Observou-se, assim, um baixo índice do exame físico da mama, exatamente este que deveria ser rotina da consulta médica nos ambulatórios. No que tange aos hábitos gerais de saúde, $55 \%$ da amostra se alimenta de comidas gordurosas, conhecido fator de risco ao câncer de mama, e $29 \%$ da amostra é de fumantes, igualmente fator relacionado de risco ao câncer de mama.

\section{Instrumento de Coleta de Dados}

O instrumento utilizado foi uma entrevista estruturada constituída de duas partes: (a) conhecimentos gerais das mulheres sobre o câncer de mama, o auto-exame da mama e hábitos gerais de saúde; (b) levantamento das vantagens e desvantagens em praticar o auto-exame da mama para fins de identificação das crenças comportamentais e normativas modais salientes, segundo o modelo postulado pelos autores da Teoria da Ação Racional (Fishbein \& Ajzen, 1975). As entrevistas foram realizadas individualmente não havendo recusa por parte das respondentes em participar da pesquisa.

Após realizadas as entrevistas, os procedimentos adotados com os dados coletados foram os seguintes: 1) para identificar as crenças e referentes modais salientes emitidos pela amostra, foi adotado o critério de escolher todas as crenças, cuja freqüência mínima fosse acima de seis, o que correspondeu a um percentual superior a $75 \%$ do total das crenças eliciadas; 2) pedir a cinco juízes (dois doutores em psicologia e três alunos de pós-graduação) para agruparem as respostas em categorias comuns, através de uma análise de conteúdo, e finalmente; 3) computar as frequiências das crenças emitidas para o comportamento determinado.

\section{Resultados e Discussão}

Crenças Comportamentais Modais Salientes relativas ao comportamento "Praticar o auto-exame da mama":

Considerando os critérios anteriormente mencionados, foi obtido um total de 129 crenças comportamentais modais salientes, de 
um total de 132 crenças emitidas. Essas crenças foram categorizadas, de acordo com as apreciações feitas pelos juízes, em nove dimensões. De uma forma em geral, a amostra pesquisada percebeu mais vantagens do que desvantagens em relação à prática do auto-exame da mama. Das nove dimensões, seis são de crenças positivas (1, 2, 3, 4,7 e 9) e três são de crenças negativas (5, 6 e 8). A Tabela 3 apresenta as dimensões encontradas.

Tabela 3

Crenças comportamentais modais salientes respondidas pela amostra $(N=40)$ em relação à pergunta: "Quais são para você as vantagens $e$ desvantagens de praticar o Auto-exame da Mama?"

\begin{tabular}{lcr}
\hline Crenças Comportamentais & Freqüência & $\%$ \\
Modais Salientes & & \\
& 23 & 17,42 \\
\hline 1. Não ver nenhuma desvantagem & 21 & 15,90 \\
2. Encontrar nódulos (caroços) & 21 & 15,90 \\
3. Evitar o câncer de mama & 21 & 15,90 \\
4. Cuidar da saúde & 11 & 8,33 \\
5. Confiança médica & 9 & 6,82 \\
6. Incapacidade de fazer o auto-exame & 8 & 6,10 \\
7. Se sentir melhor & 8 & 6,10 \\
8. Temor à doença & 7 & 5,30 \\
9. Evitar hospitais & & \\
10. Outras crenças idiossincráticas com & 3 & 2,27 \\
$\quad$ freqüência igual ou abaixo de 6 & & \\
\hline Total de Crenças Modais Salientes & $129 *$ & 100,00 \\
emitidas & 132 & \\
Total de crenças emitidas & & \\
\hline
\end{tabular}

* Este valor corresponde a $97,8 \%$ do total das crenças emitidas.

Avaliando-se individualmente as categorias de crenças comportamentais, verifica-se que:

(a) na categoria "Não ver nenhuma desvantagem", é possível que a falta de informação e o baixo nível de escolaridade tenham favorecido este tipo de resposta; 
(b) a categoria "Encontrar nódulos" ou "lãndras", como muitas chamavam, relaciona-se com as mensagens veiculadas pela mídia, geralmente alertando para esta vantagem do exame;

(c) a categoria "Evitar o câncer de mama" vai no mesmo sentido da anterior;

(d) a categoria "Cuidar da saúde" teve uma freqüência bastante significativa em termos de benefícios de praticar o auto-exame;

(e) a categoria "Confiança médica" respalda-se no papel do médico como detentor de conhecimentos e habilidades relacionados a saúde, sendo pois, caracterizada como uma crença negativa, uma vez que enquanto a mulher prefere que o médico a examine, ela não desempenha o comportamento, podendo não detectar o câncer precocemente;

(f) a categoria "Incapacidade em fazer o auto-exame da mama", da mesma forma que a anterior, é uma crença negativa, em que as mulheres acham o exame difícil de fazer, não sabendo diferenciar alterações próprias da mama de nódulos mamários;

(g) a categoria "Se sentir melhor" aparece como um efeito psicológico de que ao se tocar e não descobrir nada, dá uma "sensação de alívio" e a pessoa sente-se melhor;

(h) a categoria "Temor à doença", implica no estigma da doença como algo sinônimo de "morte";

(i) a categoria "Evitar hospitais" foi relacionada ao contexto do sistema público de saúde brasileiro, com filas enormes, demora em marcar consultas etc.

Algumas das crenças comportamentais encontradas são semelhantes aos achados de pesquisas anteriores, tais como, a "Incapacidade de fazer o exame" (Heyman, Tyner, Phipps \& Owen, 1991; Yoshioca \& de Souza, 1994; Stillman, 1977 e Huguley Jr. \& Brown, 1981); o "Temor à doença" (Heyman et al., 1991; Yoshioca \& de Souza, 1994); e "Encontrar nódulos" (Ronis \& Kaiser, 1989; Hill et al., 1985). A crença na "Confiança médica", diferencia-se dos achados de D'Amorim et al. (1992), estes autores encontraram que o auto-exame da mama teria a vantagem de "Suprir a falta de exame médico", como também, encontraram crenças relacionadas a questões estéticas do tipo: "Causa deformações nos seios" e "Causa flacidez 
nos seios". Estes tipos de crenças não foram encontradas no presente estudo.

Crenças Normativas Modais Salientes relativas ao comportamento "Praticar o auto-exame da mama:

Segundo a Teoria da Ação Racional (Fishbein \& Ajzen, 1975), a presença de um tipo de rede de apoio das pessoas significativas sobre o comportamento a ser desempenhado, influencia sua intenção. Foi obtido um total de 160 crenças normativas modais salientes, de um total de 166 crenças emitidas. Essas crenças foram categorizadas em quatro dimensões.

\section{Tabela 4}

Crenças Normativas Modais Salientes respondidas pela amostra $(N=40)$ em relação à pergunta: "Quais as pessoas cuja opinião sobre esta questão você considera importantes?"

\begin{tabular}{lll}
\hline $\begin{array}{l}\text { Crenças Normativas } \\
\text { Modais Salientes }\end{array}$ & Freqüência & $\%$ \\
& & \\
\hline 1. Meios de comunicação & 59 & 35.54 \\
2. Família extensa & 53 & 31,93 \\
3. Companheiros & 28 & 16,87 \\
4. Amigas & 20 & 12,05 \\
5. Outras crenças idiossincráticas com & & \\
$\quad$ frequêencia igual ou abaixo de 6 & 6 & 3,61 \\
\hline Total de Crenças Modais emitidas & $129 *$ & 100,00 \\
Total de crenças emitidas & 166 & \\
\hline
\end{tabular}

* Este valor corresponde a 96,4\% do total das crenças emitidas.

Analisando-se a Tabela 4, pode-se observar que a primeira categoria a dos "Meios de comunicação" obteve a maior frequiência, constatando-se que as campanhas públicas são vistas pela população, e que devem possuir mensagens persuasivas que atinjam realmente essas crenças, tentando modificá-las em uma direção mais preventiva. As demais categorias, "Família extensa", "Companheiro" e "Amigas", intensificam a visão de que não basta apenas motivar a mulher 
154 S. C. de M. Gonçalves e M. R. Dias

a praticar o auto-exame da mama, mas se faz necessário, sensibilizar sua rede de apoio social (família, marido, amigas) sobre a importância do exame. Com exceção da primeira categoria, todas as outras foram encontradas no estudo de D'Amorim et al. (1992).

Um fato a destacar é que, a presença do médico como estimulador do auto-exame da mama, não apareceu nesse estudo, significando dizer que os profissionais de saúde devem se conscientizar de que, após o exame físico das mamas, devem recomendar e orientar as mulheres à prática do auto-exame da mama.

\section{Conclusão}

O presente estudo, de forma geral, mostrou que, semelhantemente à literatura pesquisada, o baixo índice de prática do auto-exame da mama nas mulheres está associado às crenças comportamentais e normativas que permeiam o universo cognitivo dessas mulheres e dificultam sua realização. Foi constatado também que, em nossa amostra, "mulheres de baixa renda", a falta de informação, o nãoincentivo dos profissionais de saúde e a percepção negativa da doença, contribuem para o surgimento e permanência dessas crenças.

Outra crença relevante encontrada foi a "Incapacidade de fazer o auto-exame", ou seja, sentir-se competente é relevante para essas mulheres aderirem à essa prática (Celentano \& Holtzman, 1983; Rutledge, 1987), e para isso, programas educacionais, do tipo "oficinas de trabalho" que ensinem as mulheres a fazerem o auto-exame e a desenvolverem habilidades são eficazes (Amorim, Batista, Primo \& Cabalini, 1998). Seidl e Gimenes (1997) chamam a atenção para este fato afirmando que, "pessoas que não se sentem suficientemente esclarecidas e que não tem confiança na sua habilidade em executar o auto-exame, provavelmente, não vão aderir a essa prática" (p.284).

Segundo Maurer (1997), sabendo-se que as mulheres são influenciadas por seus referentes ou rede de apoio, seria interessante que esses programas incorporassem um tipo de "educação de pares" que tentassem estabelecer o auto-exame da mama como uma rotina normal de saúde nas mulheres, principalmente nas mais jovens. Na visão de Wagle, Komorita e Lu (1997), nas mulheres mais velhas, o 
suporte social também é importante e foi associado a uma maior freqüência do auto-exame da mama.

Os conteúdos significativos aqui encontrados, servirão de indicadores para futuras campanhas preventivas do câncer de mama e à prática do auto-exame. Estudos mais detalhados sobre intenções e efeitos das comunicações persuasivas na modificação dessas crenças deverão ser feitos, visando-se um melhor entendimento da questão e, conseqüentemente, um maior número de prática do auto-exame e um menor índice do câncer de mama em nosso país.

\section{Referências}

Ajzen, I., \& Fishbein, M. (1980). Understanding attitudes and predicting social behavior. Englewood Clifs: Prentice Hall.

American Cancer Society (1993). Cancer Statistics 1993 (Professional Education Publication). New York: Autor.

Amorim, M. H. C., Batista, E. M., Primo, C. C., \& Cabalini, N. C. (1998). Prevenção e diagnóstico precoce do câncer, através da Oficina de Trabalho: mulher, uma viagem ao seu corpo. Trabalho apresentado no III Congresso Brasileiro de Psico-Oncologia, Goiânia, GO.

Bland, K. I., \& Copeland, E. M. (1994). A mama: tratamento compreensivo das doenças benignas e malignas. São Paulo: Manole.

Baron, C. R., Houfek, J. F., \& Foxal, M. J. (1997). Coping style, health beliefs and breast self-examination. Mental Health Nursing, 18, 331-350.

Brubaker, R.G \& Fowler, C. (1990). Encouraging college males to perform testicular self-examination: evaluation of persuasive message based on the revised Theory of Reasoned Action. Journal of Applied Social Psychology, 17, 1411-1422.

Calnan, M., \& Rutter, D.R. (1986). Do health beliefs predict health behavior? An Analysis of breast self-examination. Social Science Medicine, 22, 673-678.

Cameron, A., \& Hinton, J. (1968). Delay in seeking treatment for mamary tumours. Cancer, 21, 1121-1126.

Celentano, D. D., \& Holtzman, M.,S. (1983). Breast self-examination competency: an analysis of self-reported practice and associated characteristicis. American Journal of Public Health, 73, 1321-1323.

Champion, V. L. (1985). Use of the health belief model in determining frequency of breast self-examination. Research in Nursing and Health, 8, 373-379. 
156 S. C. de M. Gonçalves e M. R. Dias

Costa, D., Mogos, I., \& Toma, T. (1985). Eficacy and safety of mianserin in the treatment of depression of women with cancer. Acta Psychiatric, 72 (320), 85-92.

D'Amorim, M. A., \& Gomide, S. (1986). Comportamento Sexual pré-marital: um teste da Teoria da Ação Racional. Psicologia: Teoria e Pesquisa, 2, 32-38.

D’Amorim, M. A, Freitas, C. M., \& de Sá, G.A. (1992). A motivação para realizar o auto-exame da mama: uma aplicação da Teoria da Ação Planejada. Psicologia: Teoria e Pesquisa, 8, 169-185.

Dela Coleta, M. F. (1992). Características biográficas e cognitivas relacionadas à prevenção do câncer em mulheres. Trabalho apresentando no $2^{\circ}$ Encontro Brasileiro de Psico-Oncologia, Brasília, DF.

Dias da Costa, J. S. D, Piccini, R. X., \& Moreira, M. R. (1995). Avaliação da prática do auto-exame e exame físico das mamas na cidade de Pelotas - RS. Revista Brasileira de Ginecologia e Obstetrícia, 17, 621-632.

Dias, M. R. (1995). AIDS, comunicação persuasiva e prevenção: uma aplicação da Teoria da Ação Racional. Tese de Doutorado não-publicada, Universidade de Brasília, Brasília.

Edwards, V. (1980). Changing breast self-examination behavior. Nursing Research, 29, 301-306.

Fishbein, M. \& Ajzen, I. (1975). Belief, attitude, intention and behavior: an introduction to theory and research. Reading. Massachussets: Adison - Wesley.

Fishbein, M. (1990). Factores que influyen en la intencion de estudiantes en decir a sus parejas que utilicen condom. Revista de Psicologia Social y Personalidad, 6, 1-2.

Foster Jr., R. S., Lang, S. P., Costanza, M. C., Worden, J. K., Maines, C. R. \& Yates, J.W. (1978). Breast self-examination practice and breast-cancer stage. The New England Journal of Medicine, 299, 265-270.

Freitas Jr, R., Soares, V. F., Melo, N. F., Andrade, M. L., \& Philocréon, G. R. (1996). Fatores determinantes do conhecimento e prática do auto-exame da Mama. Revista Brasileira de Ginecologia e Obstetrícia, 18, 387-391.

Greenwald, P., Nasca, P. C., \& Lawrence, C. E. (1978). Estimated effect of breast self-examination and routine physician examinations on breast-cancer mortality. New England Journal of Medicine, 299, 271-273.

Heyman, E., Tyner, R., Phipps, C., Cave, L., \& Owen, D.C. (1991). Is the hospital setting that place for teaching breast selfexamination?. Cancer Nursing, 14, 35-40. 
Hill, D., Gardner, G., \& Rassaby, J. (1985). Factors predisposing women to take precaution against breast and cervix cancer. Journal of Applied Social Psychology, 15, 59-79.

Howe, H. L. (1981). Social factors associated with breast selfexamination among high risk women. American Journal of Public Health, 71, 251-255.

Huguley Jr., C. M., \& Brown, R. L. (1981). The Value of breast selfexamination. American Cancer Society, 47, 989-995.

Lierman, L. M., Young, H. M., Kasprzyk, D., \& Benoliel, J.Q. (1990). Predicting breast self-examination using the Theory of Reasoned Action. Nursing Research, 39 (2), 97-101.

Lierman, L. M., Young, H., Powell-Cope, G., Gergiadou, F., \& Benoliel, J. Q. (1994). Effects of education and support on breast selfexamination in older women. Nursing Research, 43 (3), 158-163.

Lopes, E. R.; Rebelo, M. S., Adib, A. R., \& Abreu, E. (1996). Câncer de mama: epidemiologia e grupos de risco. Revista Brasileira de Cancerologia, 42, 105-116.

Maurer, F. (1997). A peer education model for teaching breast selfexamination to undergraduate college women. Cancer Nursing, 20 (1), 49-61.

Ministério da Saúde. Instituto Nacional de Câncer. (1996). Estimativa da incidência e mortalidade por câncer no Brasil. Coordenação de Programas de Controle de Câncer - Pro-Onco. RJ: Autor.

Ministério da Saúde. Instituto Nacional de Câncer. (1997). Estimativa da incidencia e mortalidade por câncer no Brasil. Coordenação de Programas de Controle de Câncer - Pro-Onco. RJ: Autor.

Ministério da Saúde. Instituto Nacional de Câncer. (1998). Estimativa da incidência e mortalidade por câncer no Brasil. Coordenação de Programas de Controle de Câncer - Pro-Onco. R.J

Ronis, D. L., \& Kaiser, M. K. (1989). Correlates of breast selfexamination in a sample of college women: analysis of linear structural relations. Journal of Applied Social Psychology, 13, 1068-1084.

Rutledge, D. N. (1987). Factors related to women's practice of breast self-examination. Nursing Research, 36 (2), 117-121.

Salazar, M. K. (1994). Breast self-examination beliefs: a descriptive study. Public Health Nursing, 11 (1), 49-56.

Saldanha, A.A. (1997). Determinantes primários do comportamento pedir para o parceiro usar a camisinha. Trabalho apresentado no XXVI Congresso Interamericano de Psicologia, São Paulo, SP. 
158 S. C. de M. Gonçalves e M. R. Dias

Seidl, E. M. F., \& Gimenes, M. G. (1997). A prática do auto-exame na prevenção do câncer da mama. In M. G. Gimenez. (Org.), $A$ Mulher e o Câncer. São Paulo: Editorial Psy.

Stillman, M. J. (1977). Women's health beliefs about breast selfexamination. Nursing Research, 26, 121-127.

Thomas, S. M., \& Fick, A. C. (1995). Women's health. Part II: Individual, environmental and economic factors affecting adherence to recommended screening practices for breast cancer. Journal of The Louisiana State Medical Society, 147 (4), 149-155.

Yoshioca, M. R., \& de Souza, D. (1994). Auto-exame de mama: identificação de alguns fatores que influenciam sua prática. $R e$ vista da Escola de Enfermagem, 28, 215-226.

Wagle, A., Komorita, N. I., \& Lu, Z. J. (1997). Social support and breast self-examination. Cancer Nursing, 20 (1), 42-48.

Suy-Mey C. de Mendonça Gonçalves, mestre em Psicologia Social pela Universidade Federal da Paraíba e doutoranda em Psicologia Social e do Trabalho na Universidade de Brasília, é pesquisadora do Núcleo "Aspectos Psicossociais de Prevenção e da Saúde Coletiva". Endereço para correspondência: SQSW, 304, Bloco I, apto. 311, Brasília, DF. CEP 70337-090.

E-mail: suymey@funape.ufpb.br 
Mardonio Rique Dias, doutor em Psicologia, é Professor Adjunto do Departamento de Psicologia da UFPb, coordenador do Núcleo "Aspectos Psicossociais de Prevenção e da Saúde Coletiva do Mestrado em Psicologia Social.

E-mail: mardonio@pbnet.com.br 